Scalable fabrication of immunosensors based on carbon nanotube polymer composites

This article has been downloaded from IOPscience. Please scroll down to see the full text article.

2008 Nanotechnology 19075102

(http://iopscience.iop.org/0957-4484/19/7/075102)

View the table of contents for this issue, or go to the journal homepage for more

Download details:

IP Address: 161.111.180.191

The article was downloaded on 23/09/2010 at 13:47

Please note that terms and conditions apply. 


\title{
Scalable fabrication of immunosensors based on carbon nanotube polymer composites
}

\author{
Ernest Mendoza $^{1}$, Jahir Orozco ${ }^{2}$, Cecilia Jiménez-Jorquera ${ }^{2}$, \\ Ana B González-Guerrero ${ }^{1}$, Ana Calle ${ }^{3}$, Laura M Lechuga ${ }^{3}$ and \\ César Fernández-Sánchez ${ }^{2}$ \\ ${ }^{1}$ Institut Català de Nanotecnologia, Campus Universitat Autónoma de Barcelona, \\ 08193 Bellaterra, Spain \\ ${ }^{2}$ Instituto de Microelectrónica de Barcelona, CNM-IMB (CSIC), Campus Universitat \\ Autónoma de Barcelona, 08193 Bellaterra, Spain \\ ${ }^{3}$ Instituto de Microelectrónica de Madrid, CNM-IMM (CSIC), Isaac Newton 8, \\ 28760 Tres Cantos, Madrid, Spain \\ E-mail: Ernest.Mendoza.icn@uab.es
}

Received 16 November 2007, in final form 19 December 2007

Published 29 January 2008

Online at stacks.iop.org/Nano/19/075102

\begin{abstract}
In this work we present the fabrication and characterization of immunosensors based on polystyrene (PS)-multiwalled carbon nanotube (MWCNT) composites. The electrochemical properties of the sensors have been investigated and show that the surface area is increased upon addition of the MWCNT-PS layer. Furthermore, a plasma activation process is used to partially remove the PS and expose the MWCNTs. This results in a huge increase in the electrochemical area and opens up the possibility of binding biomolecules to the MWCNT wall. The MWCNTs have been functionalized covalently with a model antibody (rabbit $\operatorname{IgG}$ ). The biosensors have been tested using amperometric techniques and show detection limits comparable to standard techniques such as ELISA.
\end{abstract}

(Some figures in this article are in colour only in the electronic version)

\section{Introduction}

The use of carbon nanotubes (CNTs) for the development of sensors has been explored for a wide variety of systems like gas sensors or biosensors [1-4]. The reasons for the interest in using CNTs for sensing applications are the high surface area that CNTs possess and the existence of unoccupied $\pi$ orbitals at the surface of the CNTs. This makes their electronic properties extremely sensitive to any molecule adsorbing to their surface [5]. The modification of the electronic properties is not selective but selectivity on the sensing event can be achieved by the correct functionalization of the CNTs [6, 7]. This is very interesting for the development of biosensors where binding enzymes, proteins, nucleic acids or antibodies to the surface of the CNTs or other nanowires like silicon results in highly sensitive/selective devices $[8,9]$.
In particular, the use of CNT-polymer composites for the development of electrochemical biosensors has been extensively investigated since the first work published over a decade ago $[10,11]$. There have been reports on the use of various polymers for the preparation of CNT composites such as nafion, polyaniline [12] or even natural polymers such as chitosan [13] for amperometric sensors [14]. The main limitation of using polymer composites for this application is that most polymers are insulating and therefore hinder electron exchange between the CNTs and any electroactive species in solution. To overcome this, there have been attempts to use conducting polymers such as poly(3,4-ethylenedioxythiophene) and polyaniline to fabricate amperometric biosensors $[12,15,16]$. However, the resulting composite material suffers from low chemical stability and not very high electrical conductivity. Other conductive polymers just show redox activity in narrow $\mathrm{pH}$ ranges, which 
limits its use as electrode materials. Furthermore, with both insulating and conducting polymers, the layer surrounding the CNTs impedes accessing the CNT walls for the correct functionalization with the appropriate biomolecules, which will be used as the recognition element of the biosensor.

In this work, a novel approach for the fabrication of polymer-CNT based biosensors is presented. The polymerCNT composite is drop cast on the top of microfabricated electrodes. The resulting composite surface appears to be covered by a polymer layer surrounding the CNT, which is partially removed by a plasma treatment. The main advantages of our approach are that the CNTs are not electrically insulated and their walls are accessible for the appropriate functionalization. Moreover, our process is fully compatible with microelectronic fabrication technology and therefore the devices can be batch processed. Mechanical polishing strategies are commonplace in this field but they are not compatible with microelectronic processes and are more difficult to apply with electrodes of planar configuration [17]. Here, covalent immobilization of appropriate immunochemical receptors to the surface of CNTs enables the development of immunosensor platforms. Their performance is tested with rabbit immunoglobulin $\mathrm{G}(\mathrm{IgG})$, chosen as the model analyte. The sensitivity of these electrochemical immunosensors is comparable with that achieved with routine immunochemical techniques such as enzyme-linked immunosorbent assays (ELISAs).

\section{Experimental details}

The gold microelectrodes (100 nm Au/20 nm Ti) with an area of $1.63 \mathrm{~mm}^{2}$ were fabricated using standard $\mathrm{Si} / \mathrm{SiO}_{2} /$ metal microelectronic technology. Carboxylic-functionalized multiwalled carbon nanotubes (COOH-MWCNTs) were purchased from Nanocyl (NC-3101) and used as-received. According to the supplier specifications the MWCNTs are around $10 \mathrm{~nm}$ in diameter and according to XPS measurements contain $\leqslant 4 \%$ $\mathrm{COOH}$ groups. The preparation of MWCNT-polymer composite was carried out as follows. Polystyrene pellets (SigmaAldrich) were dissolved in toluene. Then, the MWCNTs were added to the solution and sonicated for $30 \mathrm{~min}$. A drop of the resulting solution, typically $20 \mu \mathrm{l}$, was placed onto the microelectrode surface and allowed to dry in air. The plasma etching of the polymer-MWCNT composite was performed using a Plasmalab 80 Plus Oxford Instruments RIE system. The pressure of the chamber during the process was 75 mTorr using $\mathrm{N}_{2}$ as the carrier gas and $10 \mathrm{sccm}$ of $\mathrm{O}_{2}$. The RF power was typically of $25 \mathrm{~W}$, which resulted in $\sim 100 \mathrm{~V}$ of self-bias. The surface morphology of the samples was examined using a Hitachi S4000 Field Emission Gun scanning electron microscope (SEM) operated at $15 \mathrm{kV}$.

The covalent immobilization of the antibodies or antigens to the carboxylic groups of the MWCNTs was performed using carbodimide/succinimide chemistry (EDC-NHS), as reported elsewhere [18]. Basically, the COOH-MWCNTs were incubated in a solution containing a 4:1 molar ratio of 1-ethyl3-[3-dimethylaminopropyl]carbodiimide hydrochloride (EDC) and $N$-hydroxysuccinimide-ester (NHS-ester). Afterwards, the activated carboxylic groups were left to react with rabbit IgG (Sigma, $10 \mu \mathrm{g} \mathrm{ml}^{-1}$ solution prepared in $0.1 \mathrm{M}$ phosphate buffer $\mathrm{pH} 7.2(\mathrm{~PB}))$ at $4{ }^{\circ} \mathrm{C}$ overnight. Thus, the activated carboxylic groups reacted with terminal amino groups of the antibody giving rise to stable peptide bonds. Afterwards, the modified electrodes were incubated in PB solution containing $1 \%$ bovine serum albumin (BSA) for $15 \mathrm{~min}$ and finally in a $10 \mu \mathrm{g} \mathrm{ml}^{-1}$ solution of anti-rabbit IgG (anti-IgG) labelled to horseradish peroxidase (anti-IgG-HRP) in PB for $1 \mathrm{~h}$. Washing steps were performed in between incubations using a PB solution containing $0.1 \%$ Tween 20 . The non-covalent adsorption of the $\operatorname{IgG}$ for the comparative experiments was performed following the same steps but without the addition of the EDC and NHS. For the selectivity studies, an electrode with non-labelled anti-IgG (Sigma, $10 \mu \mathrm{g} \mathrm{ml}^{-1}$ solution prepared in $0.1 \mathrm{M} \mathrm{PB}, \mathrm{pH}$ 7.2) was prepared using the same covalent binding protocol as above.

Electrochemical experiments were performed at room temperature using a type III $\mu$-Autolab potentiostat (Ecochemie), controlled with GPES 4.7 (general purpose electrochemical system) software package. Measurements were carried out with a standard three-electrode electrochemical cell that comprised a Pt counter electrode, a $\mathrm{Ag} / \mathrm{AgCl} / 10 \%(\mathrm{w} / \mathrm{v}$ ) $\mathrm{KNO}_{3}$ reference electrode (Orion) and the MWCNT-based working electrode described above. The recording of the analytical signal was carried out by dipping the sensor in a $0.1 \mathrm{M}$

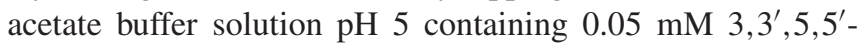
tetramethylbenzidine (TMB) and $1 \mathrm{mM} \mathrm{H}_{2} \mathrm{O}_{2}$ enzyme substrates.

\section{Results and discussion}

The MWCNT-polystyrene (PS) composite suspended in toluene is drop cast onto gold microfabricated electrodes. A SEM image of the surface of the MWCNT-PS composite (30 wt \% MWCNT/70 wt \% PS) can be observed in figure 1(a). The MWCNT content of the composites is well above the percolation threshold for this kind of material, which is around 4 wt\% [19]. The image shows the surface of the MWCNTPS composite consisting of a web of MWCNTs coated with a thick PS layer. The coating is homogeneous and the resulting nanostructures have diameters ranging from approximately 50 to $110 \mathrm{~nm}$. It is worth noticing that according to our transmission electron microscope (TEM) observations and to the specifications of the supplier, the MWCNTs are around $10 \mathrm{~nm}$ in diameter. Therefore, the image indicates that the MWCNTs are coated with a thick PS layer, between 40 and $100 \mathrm{~nm}$, which on one hand acts as a binder material giving structural integrity to the composite, but on the other hand isolates the surface of the MWCNT. For this reason, the composite was treated with a mild oxygen plasma for $1500 \mathrm{~s}$. This treatment has the objective of partially removing the PS layer from the surface of the MWCNTs and exposing their surface while keeping the binding properties of PS in order to give structural integrity to the MWCNT mat. Stronger plasma treatments may result in the detachment of MWCNTs from the surface of the sensor leading to a loss of both signal and reproducibility. The resulting surface after the 


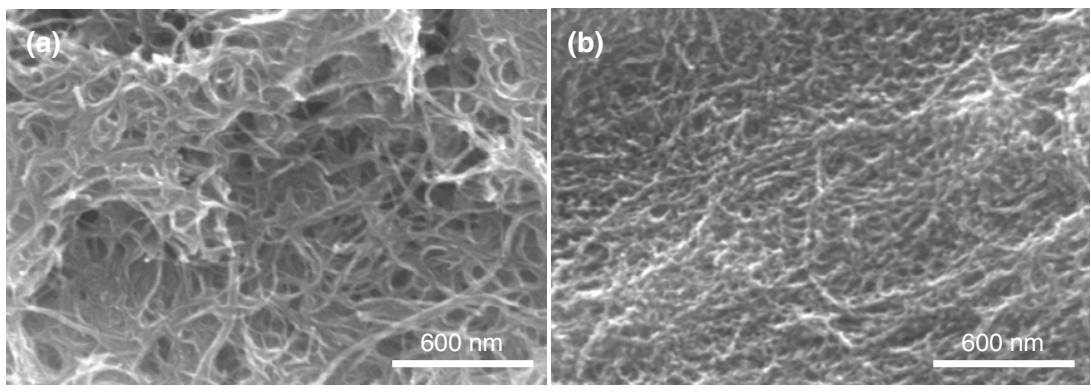

Figure 1. (a) SEM image of the surface of the pristine MWCNT-PS composite. (b) SEM image of the surface of the MWCNT-PS composite after plasma treatment for $1500 \mathrm{~s}$.
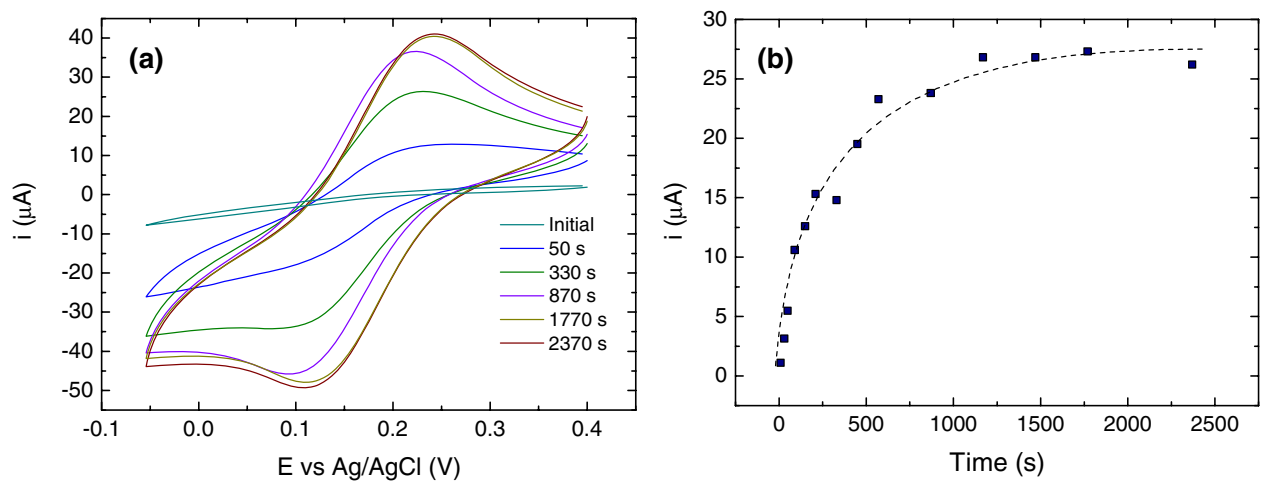

Figure 2. (a) Cyclic voltammograms for the MWCNT-PS composites at different plasma treatment times. (b) Evolution of the cathodic peak current value at $+100 \mathrm{mV}$ as a function of the plasma treatment time. Voltammograms recorded in $1.0 \mathrm{mM} \mathrm{K}_{3} \mathrm{Fe}\left(\mathrm{CN}_{6} / 0.1 \mathrm{M} \mathrm{KNO}_{3}\right.$, at $50 \mathrm{mV} \mathrm{s}^{-1}$.

plasma treatment can be observed in figure 1(b). Clearly, the surface of the MWCNT-PS composite is strongly modified by the plasma treatment by partially removing the polymer and exposing the surface of the MWCNTs. After the treatment, the MWCNTs are visible and partially covered with some polymer, which binds them in contrast with the previous fully coated MWCNTs. The power of the plasma treatment was kept low (25 W RF, -100 V self-bias) compared to other studies [20,21] in order to avoid defect formation on the MWCNTs and keep the stoichiometry of the carboxylic group. However, it is possible that some extra carboxylic groups could be introduced with such a treatment. In any case, the number of extra carboxylic groups cannot be large because control experiments performed with MWCNTs that do not contain these groups did not show a detectable degree of functionalization after the plasma treatment.

The electrochemical properties of the MWCNT-PS composite and the effect of the plasma treatment have been studied by cyclic voltammetry using ferricyanide as a redox probe. Figure 2(a) shows the evolution of the cyclic voltammogram as a function of the oxygen plasma treatment for a $30 \mathrm{wt} \% \mathrm{MWCNT} / 70 \mathrm{wt} \%$ PS composite. The value of the current at $+100 \mathrm{mV}$ (versus a $\mathrm{Ag} / \mathrm{AgCl}$ reference electrode) with respect to the plasma treatment duration is shown in figure 2(b). Clearly, the voltammogram for the pristine PS-MWCNT composite shows that the electrode surface is passivated, that is, MWCNTs are isolated, so that the faradaic current recorded is negligible. Only after a
$50 \mathrm{~s}$ plasma treatment could the faradaic current, related to the reduction/oxidation of the ferricyanide redox process, be recorded. At this point, the charge transfer kinetics is still very slow, which suggests that a high degree of electrical insulation of the MWCNTs still exists. Subsequent plasma treatments lead to a dramatic increase of the current up to 25 times and also to a well defined ferricyanide voltammetric process. This indicates fast electron transfer kinetics between electrode and solution and an increase of the electrochemically active area. The peak current of the ferricyanide redox process stabilizes at around $1500 \mathrm{~s}$ when most of the MWCNT surface is exposed. Further plasma etching involved removing of MWCNTs from the surface, thus exposing the ones below, but it does not improve the electrochemical properties of the composite material. The cathodic peak current of the ferricyanide process is directly proportional to the electrochemically active area. Using the Randles-Sevcik equation [22] an estimation of the electrochemically active area after a 10 and $1500 \mathrm{~s}$ plasma treatment was carried out and the value of the roughness factor calculated. These were $0.5 \mathrm{~mm}^{2}$ and $12.4 \mathrm{~mm}^{2}$, respectively. These values indicate that the active area has increased 24-fold, this being the value of the estimated roughness factor. The potential difference between the anodic and cathodic process of the redox probe $\left(\Delta E_{\mathrm{p}}\right)$ is around $100 \mathrm{mV}$. The theoretical Nernstian value for the ferricyanide single-electron redox process is $59 \mathrm{mV}$; however, similar values to the one obtained here were previously reported using CNT-based electrodes were the nanotubes were randomly dispersed directly on a 


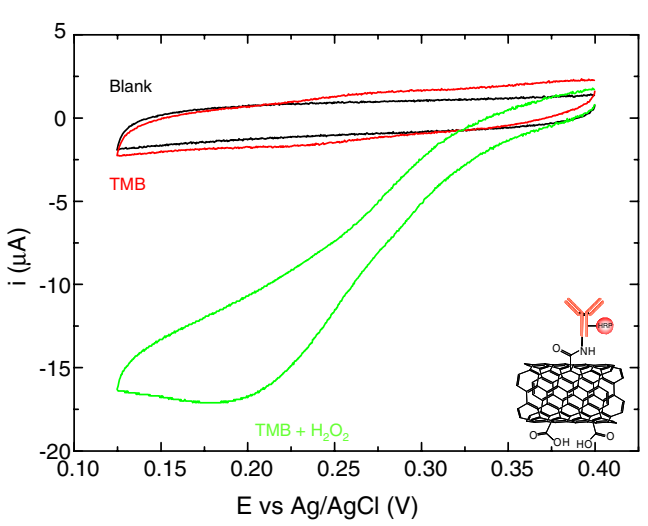

Figure 3. Cyclic voltammogram for a MWCNT-PS composite after plasma activation and the covalent immobilization of anti-IgG-HRP at the surface. The blank corresponds to the voltammogram without TMB and $\mathrm{H}_{2} \mathrm{O}_{2}$, the voltammogram in presence of TMB is also represented. Finally, the reduction of TMB in presence of $\mathrm{H}_{2} \mathrm{O}_{2}$ is depicted. Voltammograms were recorded in $0.1 \mathrm{M}$ acetate buffer solution at $\mathrm{pH} 5$ and $5 \mathrm{mV} \mathrm{s}^{-1}$.

surface or being part of a composite material. A factor related to the difference in the conduction/semiconduction properties between the walls and the ends of the carbon nanotubes appear to play an important role in the overall electrochemical behaviour of the resulting transducer [23].

The analytical system chosen for the fabrication of an immunosensor is rabbit IgG/anti-rabbit IgG. Firstly, the electrodes were modified with anti-IgG HRP conjugate in order to test the covalent conjugation protocols and the number of active sites of the MWCNTs. The HRP catalyses the oxidation of 3,3',5,5'-tetramethylbenzidine (TMB) in the presence of $\mathrm{H}_{2} \mathrm{O}_{2}$ following the overall reaction [24]:

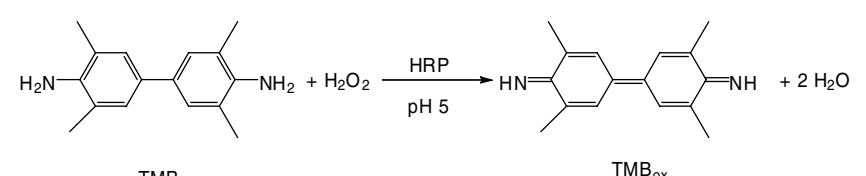

The TMB substrate is commonly applied in ELISAs based on the use of HRP label and the detection of the blue colour intensity of $\mathrm{TMB}_{\mathrm{ox}}$ by absorbance at $405 \mathrm{~nm}$ [25]. However, the redox properties of TMB enable the electrochemical detection of the catalytic reaction [26]. Indeed, the oxidation process of TMB to give the diimine derivative $\left(\mathrm{TMB}_{\mathrm{ox}}\right)$ follows two separate $1 \mathrm{H}^{+}$, single-electron processes resulting in the initial formation of a semiquinone-imine cation radical species $\left(\mathrm{TMB}^{+\cdot}\right)$ that is further oxidized to the more stable diimine derivative $\left(\mathrm{TMB}_{\mathrm{ox}}\right)$. These two processes are easily recorded by cyclic voltammetry using the CNT composite electrode developed in this work. They show half-wave potentials $\left(E_{1 / 2}\right)$ of +230 and $+420 \mathrm{mV}$, in $0.1 \mathrm{M}$ acetate buffer $\mathrm{pH} 5$.

Figure 3 shows the cyclic voltammograms of the first redox process of TMB recorded with a MWCNT-PS electrode after a $1500 \mathrm{~s}$ plasma treatment that was further functionalized with the anti-IgG-HRP.

The blank voltammogram corresponds to the signal recorded with the functionalized electrode in $0.1 \mathrm{M}$ acetate buffer $\mathrm{pH} 5$ without $\mathrm{H}_{2} \mathrm{O}_{2}$ or TMB. No faradaic processes are observed. When TMB is added to the solution, a reversible signal related to the oxidation/reduction of this electroactive molecule (TMB/TMB ${ }^{+\cdot}$ ) is shown, as described above. Following the addition of $\mathrm{H}_{2} \mathrm{O}_{2}$ to the buffer solution, another voltammetric experiment was carried out that shows a large cathodic current. This corresponds to the reduction of the oxidized form of TMB generated by the HRP catalytic reaction. The overall reaction taking place at the electrode surface can be outlined as follows:

$$
\mathrm{TMB}^{+\cdot}+1 \mathrm{H}^{+}+1 e^{-} \underset{\mathrm{HRP}}{\stackrel{\text { electrochemical }}{\rightleftarrows}} \mathrm{TMB} .
$$

The cathodic current recorded is around 15 times larger than that recorded in the absence of $\mathrm{H}_{2} \mathrm{O}_{2}$. The measurement was repeated several times with several electrodes obtaining the same signal. This demonstrates not only the high activity of HRP toward the oxidation of TMB but also the efficient and stable anchorage of the antibody-HRP conjugate on the surface of the MWCNT, and so the high density of available carboxylic groups at the composite surface.

The catalytic reaction of HRP was further followed by amperometry at a set overpotential of $+150 \mathrm{mV}$ (versus the $\mathrm{Ag} / \mathrm{AgCl}$ ref. electrode), at which all $\mathrm{TMB}$ enzymatically oxidized is reduced back to TMB.

A comparative study was performed in order to demonstrate the advantages of covalent binding versus adsorption of biomolecules to the surface of the composite electrodes. This study was also aimed at demonstrating that the plasma treatment efficiently exposes the CNT surface for the further robust immobilization of biomolecules. Adsorption processes of immunoglobulins have been commonly carried out for the fabrication of immunosensors due to their simplicity [27]. However, they are not robust enough for most applications, that is, the biomolecules come off the surface, which makes the resulting device less sensitive and reproducible. In order to carry out this study, two electrodes were incubated in an IgG solution, one activated with EDC/NHS and one without carrying out this activation step, as described in section 2. Figure 4 shows amperograms recorded using both approaches and demonstrates the great enhancement achieved when the antibody is attached to the surface by a peptide bond. The signal is amplified by a factor of 130. Therefore covalent binding of the biomolecules to the transducer increases not only the reproducibility but boosts the performance of the biosensor. Furthermore, the low signal level also indicates that the non-specific adsorption of the antiIgG-HRP to the electrode surface is also low. This suggests an effective blocking of the electrode surface by BSA.

Another important aspect in the performance of biosensors is the signal specificity. Immunoreactions are extremely selective, but unspecific binding to the surface of the sensor can lead to undesired signals. With our analytical system, the unspecific signal may come from the non-specific adsorption of the anti-IgG-HRP to the electrode surface. Figure 4 shows the amperograms recorded with two electrodes modified with IgG and with anti-IgG following the protocol described in section 2. The specific signal is given by the first electrode where the reaction between $\operatorname{IgG}$ and anti-IgG-HRP occurs. 


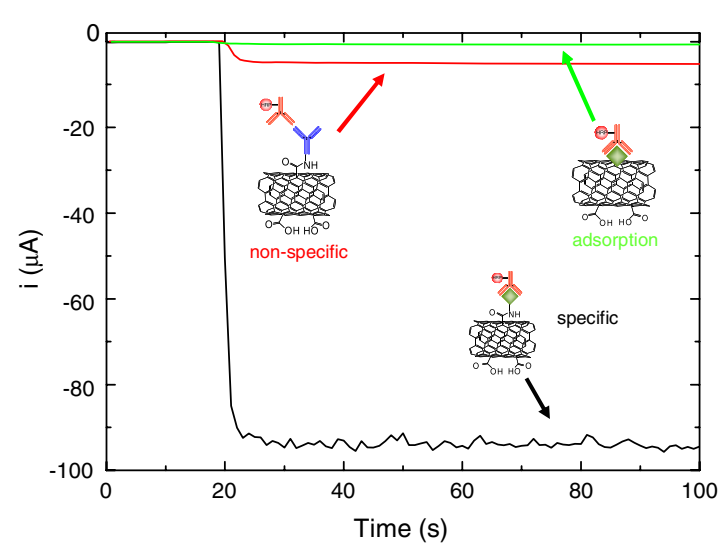

Figure 4. Amperometric signals recorded when: $\mathrm{IgG}$ is either adsorbed or covalently immobilized on the CNT walls, and is left to react with anti-IgG-HRP conjugate; anti-IgG is covalently immobilized on the CNT walls and is left to react with anti-IgG-HRP conjugate (non-specific interactions).

The signal coming from the second electrode corresponds to the unspecific signal because anti-IgG and anti-IgG-HRP should not bind together and there is just the contribution of the conjugate non-specific adsorption to the electrode surface. The relationship between the two signals gives an estimation of the signal specificity. This ratio is around $90 \%$, which indicates that the contribution of the non-specific adsorption of anti-IgG-HRP is greatly avoided. This value strongly depends on the analytical system studied and the specificity of the antibodies and could be further improved by using monoclonal antibodies. Nevertheless, although there are not many data for this area and it is difficult to compare with other analytical systems, this $90 \%$ specificity seems to be a good value compared to other works previously reported [28].

\section{Conclusions}

The fabrication of an immunosensor based on MWCNTPS composite has been presented. This process is fully scalable, repetitive and uses common techniques for the semiconductor processing industry. Thus, it can be performed at wafer level. The novelty of our approach is the controlled removal of the PS polymer from the composite surface in order to expose the surface of the MWCNT. This leads to optimum electrochemical responses of the resulting composite electrode. The removal of the polymer from the surface of the MWCNT also permits the functionalization of the MWCNT walls with the antibodies. Furthermore, we demonstrate the importance of covalently binding the biomolecules to the surface of the MWCNT in terms of sensor performance. We also show how the fabricated sensor shows a specificity in signal of $\sim 90 \%$ and since the non-specific adsorption is low, this value is controlled by the specificity of the analytical system used. Indeed, results to be published later show detection limits equivalent to conventional immunochemical techniques such as ELISA [29]. Finally, it is worth mentioning that the electrodes are potentially reusable by removing the antibody from their surface using a strong acid and repeating the plasma treatment. This type of process opens a way forward for the integration of CNTs with analytical systems based on immunoreactions such as medical devices or drug testing.

\section{Acknowledgments}

The authors thank Mr Carles Mateu for his kind assistance with the RIE equipment. E Mendoza and C FernándezSánchez acknowledge the Ministerio de Educación y Ciencia for financial support through the Programa Ramon y Cajal. This work was supported by Consolider-Ingenio 2010 (project no. CSD2006-00012).

\section{References}

[1] Kong J, Franklin N, Zhou C, Peng S, Cho J J and Dai H 2000 Science $\mathbf{2 8 7} 622$

[2] Mendoza E, Poa C H P, Henley S J, Silva S R P, Rodriguez J, Romano-Rodríguez A, Morante J R, Li Y and Zhu Y Q 2007 Carbon $\mathbf{4 5} 83$

[3] Peng H B, Chang C W, Aloni S, Yuzvinsky T D and Zettl A 2006 Phys. Rev. Lett. 9787203

[4] Lin Y, Taylor S, Li H, Shiral Fernando K A, Qu L, Wang W, Gu L, Zhou B and Sun Y P 2004 J. Mater. Chem. 14527

[5] Saito R, Dressealhaus G and Dresselhaus M G 1998 Physical Properties of Carbon Nanotubes (London: Imperial College Press)

[6] Bekyarova E, Haddon R C and Papura V 2005 Biofunctionalisation of Nanomaterials (London: Wiley-VCH)

[7] Tasis D, Tagmatarchis N, Bianco A and Prato M 2006 Chem. Rev. 1061105

[8] Patolsky F, Zheng G and Lieber C M 2006 Nanomedicine 151

[9] Wohlstadter J N et al 2003 Adv. Mater. 151187

[10] Ajayan P, Stephan O, Colliex C and Trauth D 1994 Science 2651212

[11] Schulte K, Gojny F H, Fiedler B, Sandler J K W and Bauhofer W 2005 Polymer Composites: From Nano- to Macro-Scale (New York: Springer) (chapter Carbon Nanotube-Reinforced Polymers: a State of the Art Review)

[12] Ferrer-Anglada N, Kaempgen M and Roth S 2006 Phys. Status Solidi b 2433519

[13] Tkac J, Whittaker J W and Ruzgas T 2007 Biosens. Bioelectron. 22820

[14] Wang J, Musameh M and Lin Y 2003 J. Am. Chem. Soc. 1252408

[15] Groenendal L, Joan F, Freitag D, Pielartzik H and Reynolds J R 2000 Adv. Mater. 12481

[16] Granot E, Basnar B, Cheglakov Z, Katz E and Willner I 2006 Electroanalysis 1826

[17] Chen J, Liu Z and Chen G 2007 Anal. Bioanal. Chem. 388399

[18] Grabarek Z and Gergely J 1990 Anal. Biochem. 185131

[19] Crossiord N, Loos J, Regev O and Koning C E 2006 Chem. Mater. 181089

[20] Yu J, Chen J, Deng S Z, She J C and Xu N S 2007 J. Vac. Sci Technol. B 25552

[21] Cho J H and Kim G H 2006 J. Appl. Phys. 458317

[22] Wang J 2001 Analytical Electrochemistry 2nd edn (New York: Wiley)

[23] Gooding J J 2005 Electrochim. Acta 503049

[24] Josephy P D, Elin T and Mason R P 1982 J. Biol. Chem. 2573669 
[25] Maplea L, Lathropa R, Bozicha S, Harmana W and Taceya R 2004 J. Immunol. Methods 295169

[26] Crew A, Alford C, Cowell D C C and Hart J P 2007 Electrochim. Acta $\mathbf{5 2} 5232$

[27] Fernández-Sánchez C and Costa-García A 1997 Biosens. Bioelectron. 12403
[28] Chen R J, Bangsaruntip S, Drouvalakis K A, Kam N W S, Shim M, Li Y, Kim W, Utz P J and Dai H 2003 Proc. Natl Acad. Sci. 1004984

[29] Fernández-Sánchez C, Orozco J, Jiménez-Jorquera C, González-Guerrero A B, Calle A, Lechuga L M and Mendoza E 2008 in preparation 